Cite this: Phys. Chem. Chem. Phys., 2011, 13, 17599-17605

\title{
Interaction strength between proteins and polyelectrolyte brushes: a small angle $X$-ray scattering study $\dagger$
}

\author{
Katja Henzler, ${ }^{a b}$ Björn Haupt, ${ }^{a b}$ Sabine Rosenfeldt, ${ }^{b}$ Ludger Harnau, ${ }^{c d}$ \\ Theyencheri Narayanan ${ }^{e}$ and Matthias Ballauff*af
}

Received 8th March 2011, Accepted 9th August 2011

DOI: $10.1039 / \mathrm{clcp20663j}$

We present an investigation of $\beta$-lactoglobulin adsorption onto spherical polyelectrolyte brushes (SPBs) by small angle X-ray scattering (SAXS). The SPB consists of a polystyrene core onto which long chains of poly(styrene sulfonate) are grafted. The amount and the distribution of proteins adsorbed in the brush layer at low ionic strength can be derived from SAXS. The analysis of the SAXS data reveals additionally that some of the protein molecules form aggregates of about six monomers in the adsorbed state. Furthermore, the position and the amount of slightly bound protein can be detected by the combination of the SAXS results and the SPB loading after extensive ultrafiltration. The total amount of adsorbed protein is compared to data derived from isothermal titration calorimetry. The comparison of both sets of data demonstrates that the protein molecules in the inner layers of the spherical polyelectrolyte brush are firmly bound. Proteins located in the outer layers are only weakly bound and can be washed out by prolonged ultrafiltration.

\section{Introduction}

The interaction of proteins with surfaces is of central interest in modern biotechnology. ${ }^{1-4}$ Often protein adsorption has to be prevented in order to avoid denaturation of the proteins and biofouling. ${ }^{1,2,5}$ However, in many cases the adsorption of proteins cannot be totally suppressed. For example, nanoparticles inserted into biological fluids take up proteins in many cases. This protein corona caused by protein adsorption, e.g. from blood, determines largely the biological response to the nanoparticles. ${ }^{6-9}$ Hence, an in-depth understanding of the interaction of proteins with surfaces and colloidal particles is a central problem in modern nanomedicine.

Recently, it has been shown that long polyelectrolyte chains grafted densely to a surface (polyelectrolyte brush; ref. 10) can

${ }^{a}$ Soft Matter and Functional Materials, Helmholtz-Zentrum Berlin für Materialien und Energie GmbH, Hahn-Meitner-Platz 1,

14109 Berlin, Germany.E-mail: matthias.ballauff@helmholtz-berlin.de;

Fax: + 49308062 43071; Tel: + 49308062307142308

${ }^{b}$ Physical Chemistry I, University of Bayreuth, Universitätsstr. 30, 95440 Bayreuth, Germany

${ }^{c}$ Max-Planck-Institut für Metallforschung, Heisenbergstrasse 3,

D-70569 Stuttgart, Germany

${ }^{d}$ Institut für Theoretische und Angewandte Physik, Universität Stuttgart, Pfaffenwaldring 57, D-70569 Stuttgart, Germany

${ }^{e}$ ESRF, B.P. 220, 38043 Grenoble, France

${ }^{f}$ Department of Physics, Humboldt University Berlin, Newtonstr. 15, 12489 Berlin, Germany

$\dagger$ Electronic supplementary information (ESI) available: 2-Dimensional illustrations of the protein distribution inside the brush for different protein concentrations. Furthermore, the transformation of the ITC data to an adsorption isotherm is described in more detail. See DOI: $10.1039 / \mathrm{clcp} 20663 \mathrm{j}$ bind large amounts of proteins in aqueous solution at low ionic strength. Virtually no adsorption takes place when the ionic strength of the system is high. ${ }^{11-13}$ This polyelectrolyte mediated protein adsorption (PMPA) takes place on the wrong side of the $\mathrm{pI}$, that is, at a $\mathrm{pH}>\mathrm{pI}$ where the protein and the brush layer carry the same net charge. Recent work has demonstrated that the PMPA takes place both on planar brushes, ${ }^{11,12}$ as well as onto spherical polyelectrolyte brushes (SPBs). ${ }^{13-16}$ The SPB consists of a polystyrene core (PS; diameter: $c a .100 \mathrm{~nm}$ ) onto which long chains of polyelectrolytes are grafted.

The PMPA is now well understood in terms of the counterion release: ${ }^{13,17-21}$ the protein surface exhibits positively charged patches even on the wrong side of the isoelectric point. If a protein molecule enters the polyelectrolyte layer the positive patch will act as a multivalent counterion of the negatively charged polyelectrolyte chains. Thus, a concomitant number of counterions confined in the brush layer is released. ${ }^{13,22}$ This release of counterions leads to a significant gain in entropy that can be determined by calorimetric methods. ${ }^{18}$ If the ionic strength of the system is high, this counterion release force is not operative anymore and no adsorption takes place. It should be noted that the adsorption of protein proceeds with little modification of the secondary structure. ${ }^{14,23-25}$

Up to now, the adsorption of proteins onto the SPB has been mostly studied by the ultrafiltration (UF) method: ${ }^{13}$ the protein and the SPB are mixed in a solution in which the $\mathrm{pH}$ and the ionic strength have been adjusted to a given value. Thereafter, the non-bound proteins are removed by 
ultrafiltration with an excess of aqueous buffer adjusted to the same ionic strength. Evidently, proteins which are only weakly bound to the SPB will be washed away. Here, the question arises which part of the radial brush layer binds the protein more tightly so that it cannot be washed away by UF. The binding of the protein is expected to be tighter near the surface of the core particles because of the higher density of polyelectrolyte chains.

Small-angle X-ray scattering (SAXS) is the method of choice to investigate the spatial distribution of the proteins within the spherical surface layer. ${ }^{14-16}$ Moreover, SAXS gives the total amount of proteins bound to the SPB and allows us to monitor the uptake of proteins in a time-resolved manner. ${ }^{16}$ Recently, we demonstrated that isothermal titration calorimetry (ITC) gives precise information about the adsorption isotherm and the thermodynamics of the binding of proteins to the SPB. ${ }^{18}$ Thus, we have three independent methods that allow us to monitor the uptake of proteins to the SPB: the UF method that gives only the tightly bound protein, the ITC that yields the total amount of bound proteins and SAXS that allows us to analyze the distribution of the proteins within the brush.

The aim of the present paper is a comprehensive comparison of the three different methods applied on the same brush and the protein system. The SPB used in the present study carries chains of the strong polyelectrolyte poly(styrene sulfonate). Bovine $\beta$-lactoglobulin (BLG) was used as a model protein because the uptake of BLG has been studied by ITC recently. ${ }^{18}$ The goal of the present study is twofold: we first study the distribution of the adsorbed BLG in the brush layer. The total amount of adsorbed protein derived from SAXS is compared to the one derived from ITC and UF. In a second step, we use SAXS to analyze the aggregation of protein within the brush layer. We expect BLG to aggregate in the layer for the following reasons: the strong confinement of the counterions within the layer of polyelectrolyte chains ${ }^{10}$ creates a micro-environment within the brush layer leading to a distinctly lower $\mathrm{pH}$ value in the layer. BLG is an excellent model protein because the structural transitions as a function of the $\mathrm{pH}$ value are welldocumented for this protein. ${ }^{26-31}$ In the $\mathrm{pH}$ range between 3.5 to 4 (the native $\mathrm{pH}$ of milk) $\mathrm{BLG}$ exists as a dimer. ${ }^{26}$ At $\mathrm{pH}$ values below 3.5 the BLG dimer starts to dissociate into monomeric subunits. ${ }^{26}$ In the $\mathrm{pH}$ range between 4 and 5.2 it was reported that the protein forms octamers. ${ }^{27,28}$ In addition, it was shown by Majhi et al. that the aggregation does not stop at a certain association number. ${ }^{30}$ The aggregation is distinctly stronger for the genetic variant $\mathrm{A}$ than for the $\mathrm{B}$ variant. $^{26-29}$ The variant A and B differs only at positions 64 (Asp/Gly) and 118 (Val/Ala). ${ }^{26}$ The genetic variant A of BLG has an additional carbonyl-group per monomer unit which seems to play an important role in the aggregation process. ${ }^{26}$ Above $\mathrm{pH}=5.2$ BLG forms again dimers. ${ }^{26-28}$

The paper is organized as follows. We first study the uptake and distribution of BLG in the brush layer by SAXS. Second, we compare the result of the SAXS analysis with the data obtained by isothermal titration calorimetry and by the method devised by Wittemann et al. ${ }^{32}$ The comparison of these data stemming from totally different methods is shown to give full insights into the process of protein adsorption onto the SPB.

\section{Experimental}

\subsection{Materials}

$\beta$-Lactoglobulin from bovine milk which contains the genetic variants A and B was purchased from Sigma (product number: L3908; Lot.: 106K7312) and was used without further purification. All other chemicals used in this study were of analytical grade.

\subsection{Spherical polyelectrolyte brushes (SPBs)}

The synthesis of the brush particles was done as described in ref. 13 by photo-emulsion polymerisation. The system was purified through extensive ultrafiltration against pure water. Afterwards, the $\mathrm{pH}$ was adjusted by ultrafiltration to 6.1 by $10 \mathrm{mM}$ 2-( $N$-morpholino)ethanesulfonic acid (MES) buffer. The core radius $R$ of the particles and the hydrodynamic radius of the brushes were determined by dynamic light scattering to be $45 \mathrm{~nm}$ and $120 \mathrm{~nm}$, respectively.

\subsection{Small angle X-ray scattering (SAXS)}

The SAXS experiments were performed at the beamline ID2 of the European Synchrotron Radiation Facility (ESRF, Grenoble, France). ${ }^{33}$ Samples with different BLG concentrations were prepared in MES buffer. These solutions were mixed with SPB where the SPB concentration was kept constant at $1 \mathrm{wt} \%$. The incident wavelength $\lambda$ of the X-rays was $0.1 \mathrm{~nm}$. In order to cover the whole range of the scattering vector $q,(q=$ $(4 \pi / \lambda) \sin (\theta / 2)$, with $\theta$ being the scattering angle), the SAXS intensities were recorded at two detector distances of 8 and $2 \mathrm{~m}$ by a CCD detector (FReLoN). The exposure time for both detector positions was $20 \mathrm{~ms}$. Each sample was measured ten times to improve the statistics of the scattering intensity. In order to avoid radiation damage the measurements were taken with a flow-through capillary cell.

\subsection{Adsorption isotherm obtained by ultrafiltration}

Wittemann et al. have described the experimental procedure which has been used up to now for the investigation of the adsorption behaviour by ultrafiltration. ${ }^{13,32}$ Given amounts of $\beta$-lactoglobulin were dissolved in $10 \mathrm{mM}$ MES buffer. A suspension of the SPB in MES buffer of the same ionic strength was added. All experiments were carried out at $\mathrm{pH}$ 6.1. The samples contained $1 \mathrm{wt} \% \mathrm{SPB}$ and were equilibrated for $24 \mathrm{~h}$ under gentle stirring at $4{ }^{\circ} \mathrm{C}$. Afterwards the nonbound or weakly bound protein was removed by ultrafiltration. The amount of removed protein was determined by the absorbance of the serum at a wavelength of $278 \mathrm{~nm}$ (Lambda 650 Perkin-Elmer, software UVWinLab). The amount of bound protein is the difference between the protein added to the suspension and the non-adsorbed protein.

\section{Results and discussion}

\subsection{Analysis of protein adsorption by SAXS}

In the following we describe the theoretical analysis of the SAXS data. SAXS determines the scattering intensity $I\left(q, \rho_{\mathrm{s}}, \rho_{\mathrm{p}}\right)$ as a function of the magnitude of the scattering vector $q$ and the particle number densities of the SPBs $\rho_{\mathrm{s}}$ 
and the proteins $\rho_{\mathrm{p}}$, respectively. For a system consisting of monodisperse SPBs and proteins the scattering intensity can be written as ${ }^{15}$

$$
I\left(q, \rho_{\mathrm{s}}, \rho_{\mathrm{p}}\right)=\rho_{\mathrm{s}} S\left(q, \rho_{\mathrm{s}}\right) I_{0}^{(\mathrm{s})}\left(q, \rho_{\mathrm{p}}\right)+\rho_{\mathrm{s}} I_{\mathrm{f}}(q)+\rho_{\mathrm{p}} I_{\mathrm{p}}(q),
$$

where $I_{0}^{(\mathrm{s})}\left(q, \rho_{\mathrm{p}}\right)$ describes how the scattering intensity is modulated by interference effects between radiations scattered by different parts of the same SPB. The structure factor $S\left(q, \rho_{\mathrm{s}}\right)$ is related to mutual interactions between different SPBs. Therefore, it is dependent on the degree of order of the SPBs in the sample. The contribution to the scattering intensity due to concentration fluctuations of the polyelectrolyte chains is denoted as $I_{\mathrm{f}}(q)$. In the present study, this contribution becomes important only for large scattering vectors. Finally, $I_{\mathrm{p}}(q)$ describes the scattering intensity of individual proteins or aggregates of proteins as will be discussed later.

The scattering intensity $I_{0}^{(\mathrm{s})}\left(q, \rho_{\mathrm{p}}=0\right)$ of a single SPB without added proteins is given by ${ }^{15}$

$$
I_{0}^{(\mathrm{s})}\left(q, \rho_{\mathrm{p}}=0\right)=\left(\frac{4 \pi}{q} \int_{0}^{\infty} \mathrm{d} r r \Delta b\left(r, \rho_{\mathrm{p}}=0\right) \sin (q r)\right)^{2}
$$

where $\Delta b\left(r, \rho_{\mathrm{p}}=0\right)$ is the excess (over the solvent) radial electron density profile of the SPB. Here, the origin of the spherical coordinates is taken to be the centre of the solid core of the SPB. In order to account for the core-shell structure with a homogeneous core of radius $R$ and a spatially inhomogeneous shell we use the excess electron density profile

$$
\begin{aligned}
\Delta b\left(r, \rho_{\mathrm{p}}=0\right)= & \Delta b_{\mathrm{PS}} \Theta(R-r) \\
& +\Delta b_{\mathrm{PSS}} \Theta(r-R) \exp \left(-\kappa_{\mathrm{PSS}}(r-R)^{e \mathrm{PSS}}\right) .
\end{aligned}
$$

Here, $\Theta(x)$ is the Heaviside step function and $\Delta b_{\mathrm{PS}}=$ $7 \mathrm{~nm}^{-3}$ is the excess electron density of the polystyrene core. The second term in eqn (3) with $\Delta b_{\mathrm{PSS}}=100 \mathrm{~nm}^{-3}, \kappa_{\mathrm{PSS}}=$ $0.43 \mathrm{~nm}^{-1}$ and $e_{\mathrm{PSS}}=0.68$ characterizes the electron density profile of the polyelectrolyte shell which decreases upon increasing the radial distance $r$ from the center of the SPB. The grafting nature of the poly(styrene sulfonate) shell leads to a significant smaller excess electron density $\Delta b_{\mathrm{PSS}}$ as compared to $\Delta b_{\mathrm{PSS}}^{\text {(bulk) }}=242 \mathrm{~nm}^{-3}$ of the bulk poly(styrene sulfonate) phase.

Although eqn (2) and (3) have been derived for one particle of a definite size characterized by radius $R$, in reality a range of sizes are present in the system under study, as is apparent from transmission electron microscopy micrographs of solid polystyrene core particles. Therefore, the effect of size polydispersity of the core particles is taken into account by an appropriate average using a distribution function characterizing the degree of the polydispersity (see, e.g., ref. 34). We note that the functional form of the excess electron density profile of the shell in eqn (3) reflects the size polydispersity of the grafted polyelectrolyte chains.

Within a Gaussian approximation the contribution of the concentration fluctuations of the polyelectrolyte chains reads

$$
I_{\mathrm{f}}(q)=\frac{I_{0}^{(\mathrm{f})}}{1+(q \xi)^{2}}
$$
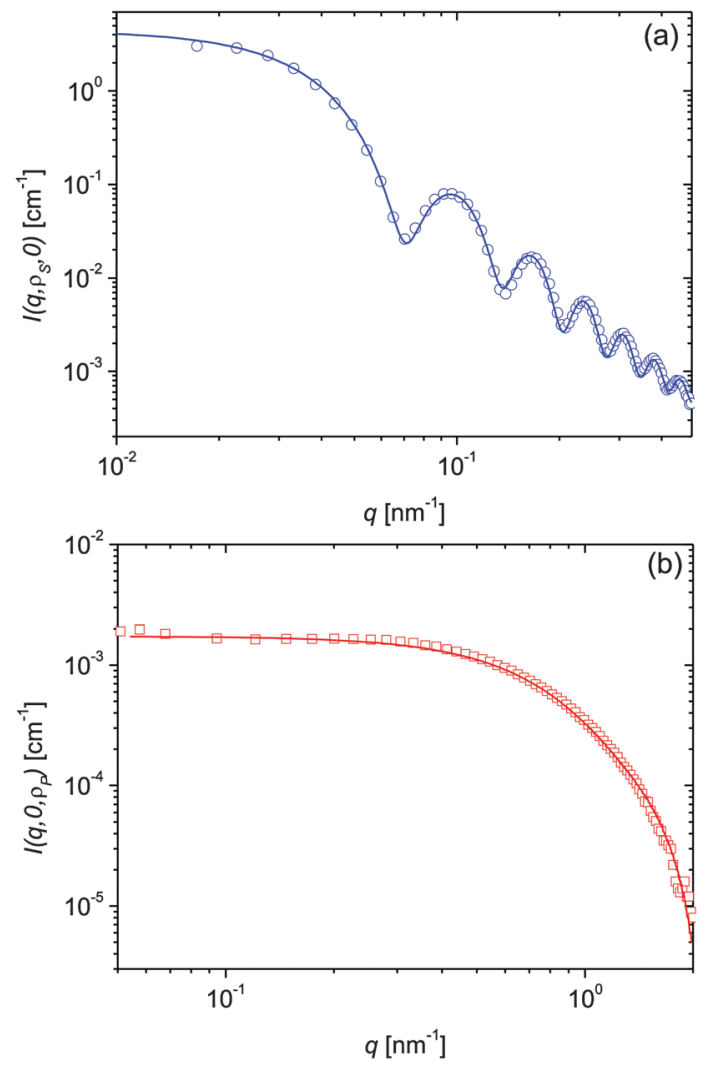

Fig. 1 (a) Measured scattering intensity $I\left(q, \rho_{\mathrm{s}}, 0\right)$ of spherical polyelectrolyte brush particles in aqueous solution (symbols). The particle number density is $\rho_{\mathrm{s}}=3.78 \times 10^{-8} \mathrm{~nm}^{-3}$. The solid line represents the result of the modeling according to eqn (1)-(4) with model parameters given in the main text. (b) Measured scattering intensity $I\left(q, 0, \rho_{\mathrm{p}}\right)$ of $\beta$-lactoglobulin (symbols) together with the calculated scattering intensity according to eqn (5). The protein number density is $\rho_{\mathrm{p}}=2.46 \times 10^{-4} \mathrm{~nm}^{-3}$.

where $\xi=2 \mathrm{~nm}$ is the correlation length ${ }^{15}$ and $I_{0}^{(\mathrm{f})}=0.00025 \mathrm{~cm}^{-1}$ determines the contribution at the vanishing scattering vector.

In Fig. 1(a) the experimental scattering intensity $I\left(q, \rho_{\mathrm{s}}, 0\right)$ for the SPBs without added proteins is compared to the calculated result for interacting SPBs with a mean core radius $R_{\mathrm{m}}=37 \mathrm{~nm}$ (solid line). Both steric and electrostatic interactions lead to a decrease of $I\left(q, \rho_{\mathrm{s}}, 0\right)$ at low scattering vectors as compared to the scattering intensity $I\left(q, \rho_{\mathrm{s}} \rightarrow 0,0\right)$ of noninteracting SPBs. We have used an integral equation theory ${ }^{35}$ in order to calculate structure factors which characterize intermolecular correlations between different SPBs. The contribution of concentration fluctuations of the polyelectrolyte chains $I_{\mathrm{f}}(q)$ becomes important for large scattering vectors $q \gtrsim 0.3 \mathrm{~nm}^{-1}$.

A comprehensive approach for modeling the scattering intensity $I\left(q, \rho_{\mathrm{s}}, 0\right)$ of a colloidal sphere with attached polymer chains has been presented by Pedersen and Gerstenberg and developed further. ${ }^{36,37}$ However, the present system is very different and contains charged chains in which the counterions present an additional component contributing to the measured scattering intensity. Moreover, it should be kept in mind that the present analysis does not aim at a description of the SPB but on the alterations effected by the uptake of protein. The latter component becomes a dominant contribution and the analysis of which can be done very well in terms of the model given in ref. 15 . 
Fig. 1(b) displays the measured and calculated scattering intensities $I\left(q, 0, \rho_{\mathrm{p}}\right)$ for free BLG. As suggested by earlier studies, BLG forms dimers at the $\mathrm{pH}$ value used in the experiment. Therefore, we use the following expression for the scattering intensity of randomly oriented noninteracting monodisperse dumbbells

$$
\begin{aligned}
I_{\mathrm{p}}(q)= & 2 \Delta b_{\mathrm{p}}^{2} V_{\mathrm{p}}^{2}\left(1+\frac{\sin \left(q L_{\mathrm{p}}\right)}{q L_{\mathrm{p}}}\right) \\
& \times\left(\frac{3}{\left(q R_{\mathrm{p}}\right)^{3}}\left(\sin \left(q R_{\mathrm{p}}\right)-q R_{\mathrm{p}} \cos \left(q R_{\mathrm{p}}\right)\right)\right)^{2} .
\end{aligned}
$$

Each dumbbell consists of two spheres with a radius $R_{\mathrm{p}}=$ $2.05 \mathrm{~nm}$ and a center-to-center distance $L_{\mathrm{p}}=3.4 \mathrm{~nm}$. Moreover, the excess electron density of BLG and the volume of the monomeric subunit are $\Delta b_{\mathrm{p}}=90.81 \mathrm{~nm}^{-3}$ and $V_{\mathrm{p}}=$ $23.25 \mathrm{~nm}^{3}$, respectively. From Fig. 1(b) it is apparent that the measured scattering intensity can be described using eqn (5).

We now turn our attention to the adsorption of BLG on SPBs. Fig. 2(a) displays the measured and calculated scattering intensities $I\left(q, \rho_{\mathrm{s}}, \rho_{\mathrm{p}}\right)$ for five different protein number densities $\rho_{\mathrm{p}}$. The uptake of BLG by the SPBs can be directly seen from the shift of the first side maximum to smaller $q$ values upon increasing the protein number density. The solid lines show the calculated results obtained from eqn (1)-(4) with

$$
\begin{aligned}
\Delta b\left(r, \rho_{\mathrm{p}}\right)= & \Delta b\left(r, \rho_{\mathrm{p}}=0\right)+\Delta b_{\mathrm{p}}\left(r, \rho_{\mathrm{p}}\right), \\
\Delta b_{\mathrm{p}}\left(r, \rho_{\mathrm{p}}\right)= & n\left(\rho_{\mathrm{p}}\right) \Theta(r-R) \Theta\left(R_{1}-r\right) e^{\left(-\sigma_{1}\left(r-R_{l}\right)^{2}\right)} \\
& +\Theta\left(r-R_{1}\right) \exp \left(-\sigma_{2}\left(r-R_{\mathrm{l}}\right)^{2}\right) .
\end{aligned}
$$

Here, $R_{1}=R+17 \mathrm{~nm}, \sigma_{1}=0.009 \mathrm{~nm}^{-2}$, and $\sigma_{2}=$ $0.0025 \mathrm{~nm}^{-2}$. The normalization constant $n\left(\rho_{\mathrm{p}}\right)$ has been chosen such that the integrated excess electron density yields the total number of electrons of adsorbed proteins. The excess electron density profile $\Delta b_{\mathrm{p}}\left(r, \rho_{\mathrm{p}}\right)$ shown in Fig. 2(b) by the dashed line demonstrates that BLG avoids a direct contact with the PS core because the value of the profile is rather small at the surface of the PS core. Hence, the grafted polyelectrolyte chains are responsible for the adsorption of the proteins. Moreover, the excess electron density decreases for distances $r>R_{1}$ due to the pronounced decrease of the monomer density of the grafted polyelectrolyte chains according to the second term in eqn (3) (see the black line in Fig. 2(b)). A cutout of an illustration of the brush with adsorbed BLG up to scale based on the electron density profiles of Fig. 2(b) is shown in Fig. 2(c). The ESI $\dagger$ shows for all investigated SAXS data in Fig. 2(a) a 2-dimensional illustration up to scale of the BLG distribution in the brush layer.

For low scattering vectors, the SAXS measurements do not resolve structural details of the size of the proteins. Therefore, the adsorbed proteins increase the electron density of the shell leading to the aforementioned shift and an increase of the first side maximum of $I\left(q, \rho_{\mathrm{s}}, \rho_{\mathrm{p}}\right)$. In addition, the scattering intensity increases at low scattering vectors upon increasing the number of adsorbed proteins. For high scattering vectors
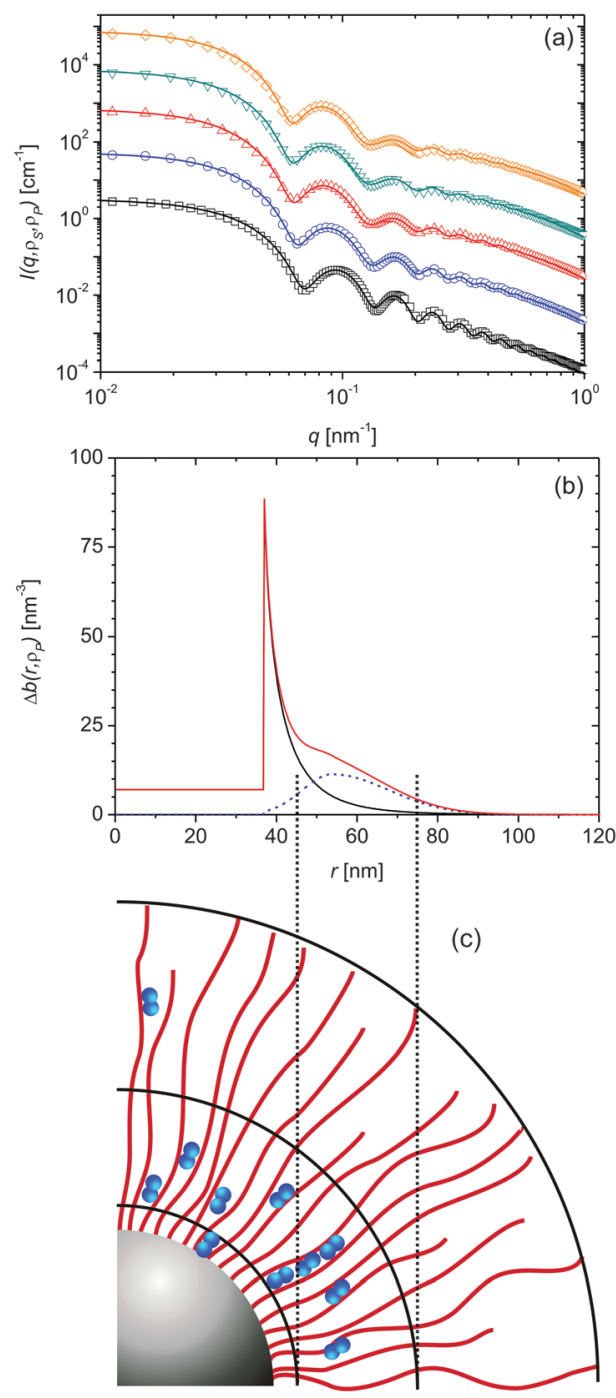

Fig. 2 (a) Experimentally determined scattering intensity $I\left(q, \rho_{\mathrm{s}}, \rho_{\mathrm{p}}\right)$ of spherical polyelectrolyte brush particles at different amounts of added $\beta$-lactoglobulin (symbols). The particle number density is $\rho_{\mathrm{s}}=$ $2.01 \times 10^{-8} \mathrm{~nm}^{-3}$, while the protein number density increases from bottom to top according to $\rho_{\mathrm{p}} / \rho_{\mathrm{s}}=1629$ (101 $\left.\mathrm{mg} \mathrm{BLG} \mathrm{g}^{-1} \mathrm{SPB}\right), 6514$ (400 mg BLG g ${ }^{-1}$ SPB), 9771 (600 mg BLG g $\left.{ }^{-1} \mathrm{SPB}\right), 12214$ (750 mg BLG g ${ }^{-1} \mathrm{SPB}$ ), 16286 (990 $\left.\mathrm{mg} \mathrm{BLG} \mathrm{g}^{-1} \mathrm{SPB}\right)$. For clarity, the upper four data sets have been shifted up. The solid lines depict the calculated scattering intensities. In panel (b) the electron density profiles of the pure brush $\left(\Delta b\left(r, \rho_{\mathrm{p}}=0\right)\right.$, black line $)$, the brush with adsorbed proteins $\left(\Delta b\left(r, \rho_{\mathrm{p}}\right)\right.$, red line $)$ and the pure adsorbed proteins $\left(\Delta b_{\mathrm{p}}\left(r, \rho_{\mathrm{p}}\right)\right.$, blue dashed line) are shown for a core radius of $37 \mathrm{~nm}$. (c) A cutout of a 2-dimensional representation of the SPB with the distribution of $400 \mathrm{mg} \mathrm{BLG} \mathrm{g}^{-1} \mathrm{SPB}$ derived from (b). The protein molecules which are in the outer part of the brush layer can be released by extensive ultrafiltration. The presented illustration is up to scale.

the resolution of the SAXS experiment is high enough to reveal the shape of individual proteins. This structural information is taken into account by the scattering intensity $I_{\mathrm{p}}(q)$ in eqn (1). It has been demonstrated that the decomposition of the scattering intensity $I\left(q, \rho_{\mathrm{s}}, \rho_{\mathrm{p}}\right)$ according to eqn (1) leads to good agreement with experimental data of SPBs and adsorbed proteins provided the scattering intensity $I_{\mathrm{p}}(q)$ of free proteins is used as an input in eqn (1). ${ }^{15,16}$ In those earlier studies the 
proteins bovine serum albumin and bovine pancreatic ribonuclease A have been used. However, in the case of BLG used in the present study the scattering intensity $I\left(q, \rho_{\mathrm{s}} \neq 0, \rho_{\mathrm{p}}\right)$ cannot be described using the scattering intensity $I_{\mathrm{p}}(q)$ given by eqn (5), although this expression and eqn (1) lead to an agreement with the measured scattering intensity $I\left(q, 0, \rho_{\mathrm{p}}\right)$ of free BLG as is apparent from Fig. 1(b). In order to obtain the scattering intensities $I\left(q, \rho_{\mathrm{s}} \neq 0, \rho_{\mathrm{p}}\right)$ given by the solid lines in Fig. 2(a) we have used the scattering intensity of aggregated dumbbells $I_{\mathrm{p}}(q)$ as an input in eqn (1). As already mentioned in the Introduction, BLG is expected to form superstructures in the brush layer because of a combination of its well-known aggregation behaviour as a function of the $\mathrm{pH}$ value and the modified $\mathrm{pH}$ value in the brush layer as compared with that of the surrounding aqueous solution. In order to justify this argumentation based on the decomposition of the scattering intensity $I\left(q, \rho_{\mathrm{s}}, \rho_{\mathrm{p}}\right)$ according to eqn (1), we have performed additional calculations by arranging $N_{\mathrm{p}}=\rho_{\mathrm{p}} / \rho_{\mathrm{s}}$ protein monomers around a solid core of radius $R$. We have considered various types of protein superstructures and we conclude that the adsorbed proteins form aggregates of about six monomers. Fig. 3 displays an example of a comparison of a measured scattering intensity (symbols) and calculated scattering intensities for aggregated proteins (blue line) as well as for protein dumbbells (magenta line). The radial distribution function of the aggregates is characterized by the functional form of the proteins excess electron density profile $\Delta b_{\mathrm{p}}\left(r, \rho_{\mathrm{p}}\right)$ given by eqn (7) and shown in Fig. 2(b). For comparison we note that the influence of SPBs on the structure of bovine hemoglobin has been discussed in ref. 14. Bovine hemoglobin forms a tetramer in solution which may dissociate inside a brush due to various intermolecular interactions, while BLG considered in the present study forms aggregates inside a brush.

Finally, it is worthwhile to mention that we have performed an additional SAXS experiment on loaded SPBs using a higher

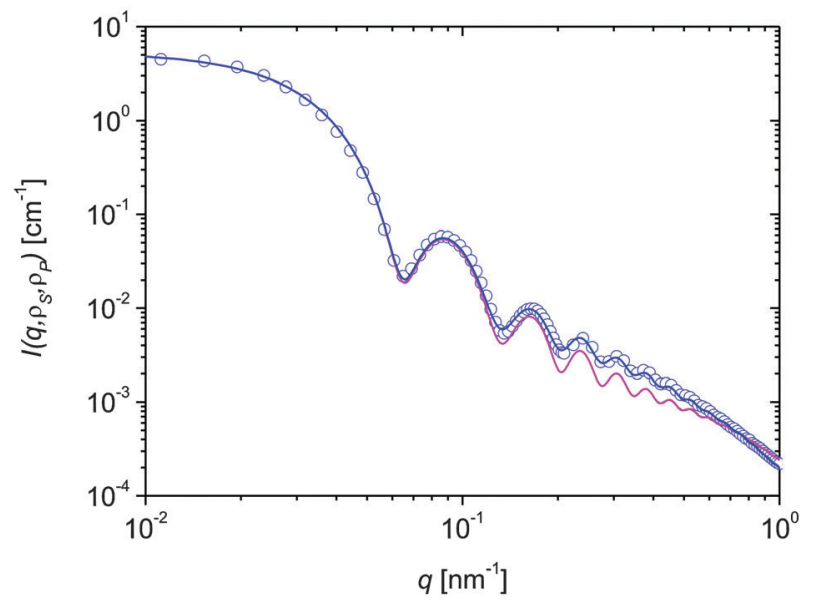

Fig. 3 Measured scattering intensity $I\left(q, \rho_{\mathrm{s}}, \rho_{\mathrm{p}}\right)$ of spherical polyelectrolyte brush particles for the protein number density $\rho_{\mathrm{p}} / \rho_{\mathrm{s}}=6514$ with $\rho_{\mathrm{s}}=2.01 \times 10^{-8} \mathrm{~nm}^{-3}$ (symbols). The magenta line displays the calculated result with the scattering intensity of protein dumbbells (see eqn (5)) as input in eqn (1), while the blue line depicts the calculated results taking into account an aggregation of protein dumbbells inside the polyelectrolyte brush.

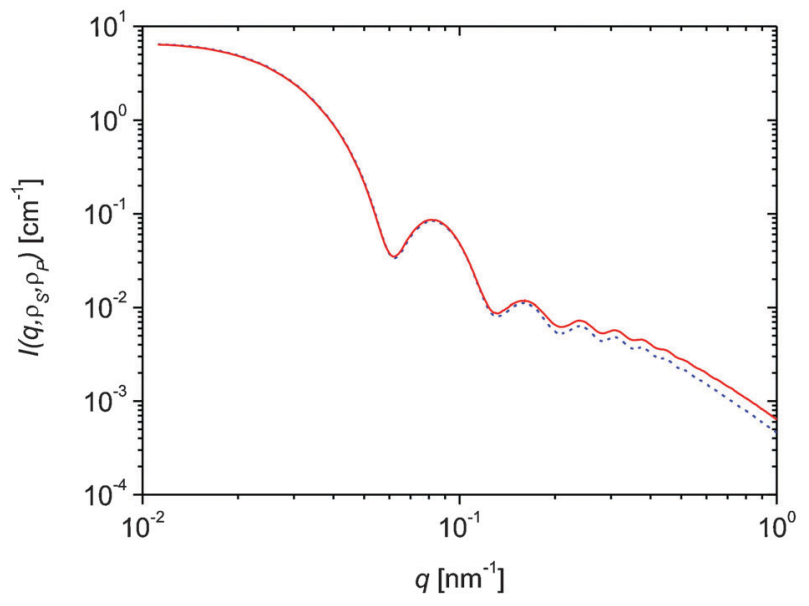

Fig. 4 Comparison of measured scattering intensities $I\left(q, \rho_{\mathrm{s}}, \rho_{\mathrm{p}}\right)$ of spherical polyelectrolyte brush particles for two high protein number densities: $\rho_{\mathrm{p}} / \rho_{\mathrm{s}}=22806$ (solid curve) and $\rho_{\mathrm{p}} / \rho_{\mathrm{s}}=16286$ (dashed curve). In both cases the particle number density is $\rho_{\mathrm{s}}=$ $2.01 \times 10^{-8} \mathrm{~nm}^{-3}$.

protein density $\left(\rho_{\mathrm{p}} / \rho_{\mathrm{s}}=22806\right)$ than the ones leading to the scattering intensities shown in Fig. 2(a). Fig. 4 displays a comparison of the measured scattering intensity $I\left(q, \rho_{\mathrm{s}}, \rho_{\mathrm{p}}\right)$ for the protein number densities $\rho_{\mathrm{p}} / \rho_{\mathrm{s}}=22806$ (solid curve) and $\rho_{\mathrm{p}} / \rho_{\mathrm{s}}=16286$ (dashed curve). For large scattering vectors $q \gtrsim 0.2 \mathrm{~nm}^{-1}$ one finds the expected increase of the scattering intensity upon increasing $\rho_{\mathrm{p}}$ according to the last term in eqn (1), while there is neither an increase of the scattering intensity at low scattering vectors nor a shift of the first side maximum in contrast to the results shown in Fig. 2(a). Therefore, it is tempting to conclude from Fig. 4 that there is no additional protein uptake into the brush layer upon increasing the protein number density from $\rho_{\mathrm{p}} / \rho_{\mathrm{s}}=16286$ to $\rho_{\mathrm{p}} / \rho_{\mathrm{s}}=22806$ because the solid and dashed curves are very similar at low scattering vectors. However, such an interpretation could be misleading because the limited scattering vector range $q \lesssim 0.01 \mathrm{~nm}^{-1}$ accessible by SAXS does not detect intermolecular correlations arising from proteins which are located close to rather stretched polyelectrolyte chains and separated by a large distance. Due to the Coulomb interaction some polyelectrolyte chains may be highly extended at the expense of conformational entropy. In order to test this hypothesis we have performed additional calculations by arranging protein monomers around a solid core. We have found an increase of the scattering intensity in the low scattering vector regime $q \in[0.001,0.01] \mathrm{nm}^{-1}$ in the case that some proteins are located in the shell at a large distance from the core. In view of the limited scattering vectors accessible by SAXS it is not possible to detect correlations between proteins which are located at the outer parts of the polyelectrolyte shell. However, these proteins contribute to the scattering intensity as individual units at large scattering vectors.

\subsection{Adsorption isotherm by ITC}

The preceding section has demonstrated that SAXS allows us to determine the total amount of adsorbed protein and its state 
of aggregation. This information can now be compared to isothermal titration calorimetry (ITC) measurements applied recently to the adsorption of BLG to SPB.${ }^{18}$ ITC measures the heat evolving when a protein solution is added to an aqueous solution of the SPB. The analysis of the adsorption of BLG to the SPB carrying a dense layer of PSS-chains showed that the adsorption enthalpy is positive. Therefore, the driving force for protein adsorption must be entropic. ${ }^{18}$

In the following we derive the total amount of the adsorbed protein from the data (further information of the following section can be found in the ESI $\dagger$ ). We apply the two sets of the independent binding sites (TSIS) model since this model was shown to give an excellent fit of the ITC data. ${ }^{18} \mathrm{~A}$ detailed discussion of the meaning of this model is given in ref. 18 . Each set of the two different binding sites has its own characteristic binding constant $\left(K_{\mathrm{A} 1}, K_{\mathrm{A} 2}\right)$, molar heat of binding $\left(\Delta H_{1}, \Delta \mathrm{H}_{2}\right)$ and number of sites $\left(N_{1}, N_{2}\right) .{ }^{38}$ It is assumed that each binding site is independent from the other. Each type of site has its own fractional saturation $\left(\Theta_{1}, \Theta_{2}\right)$. Thus, it is possible to define the binding constants for the two binding sites ${ }^{38}$

$$
K_{\mathrm{A} 1}=\frac{\Theta_{1}}{\left(1-\Theta_{1}\right)[\mathrm{BLG}]} K_{\mathrm{A} 2}=\frac{\Theta_{2}}{\left(1-\Theta_{2}\right)[\mathrm{BLG}]}
$$

where $[\mathrm{BLG}]$ is the concentration of the unbound protein. The definition of the total concentration of $\beta$-lactoglobulin $[\mathrm{BLG}]_{\mathrm{t}}$ is given by ${ }^{38}$

$$
[\mathrm{BLG}]_{\mathrm{t}}=[\mathrm{BLG}]+[\text { brush }]_{\mathrm{t}}\left(N_{1} \Theta_{1}+N_{2} \Theta_{2}\right)
$$

where [brush $]_{t}$ is the total brush concentration. Solving eqn (8) for $\Theta_{1}$ and $\Theta_{2}$, respectively, and substituting into eqn (9) result in a cubic equation if the equation is solved for [BLG] (see ESI $\dagger$ ).

The cubic equation (eqn (S4) in the ESI $\dagger$ ) can subsequently be solved for each injection in closed form if the parameters $K_{\mathrm{A} 1}, K_{\mathrm{A} 2}, N_{1}$ and $N_{2}$ are known $\left(K_{\mathrm{A} 1}=3 \times 10^{7} \pm 2 \times\right.$ $10^{7} \mathrm{~L} \mathrm{~mol}^{-1}, K_{\mathrm{A} 2}=1 \times 10^{6} \pm 0.1 \times 10^{6} \mathrm{~L} \mathrm{~mol}^{-1}, N_{1}=$ $\left.1800 \pm 100, N_{2}=10300 \pm 100\right)$. From this data the fraction of bound $\beta$-lactoglobulin and the loading of the SPB particles $\tau_{\text {ads }}$ can be determined through

$$
\tau_{\text {ads }}=\frac{\left([\mathrm{BLG}]_{\mathrm{t}}-[\mathrm{BLG}]\right) 2000 M_{M}}{[\text { brush }]_{\mathrm{t}} M_{\mathrm{brush}}}
$$

where $M_{\text {brush }}$ is the molar mass of the SPB particles and is given by

$$
M_{\text {brush }}=\left(m_{\text {core }}-m_{\text {shell }}\right) N_{\mathrm{A}}=\frac{\rho_{\mathrm{PS}} \frac{4}{3} \pi R^{3}}{w_{\text {core }}} N_{A}
$$

where $m_{\text {core }}$ is the mass of the PS core, $m_{\text {shell }}$ is the mass of the polyelectrolyte layer, $N_{\mathrm{A}}$ is the Avogadro constant, $\rho_{\mathrm{PS}}=$ $1.054 \times 10^{21} \mathrm{~g} \mathrm{~nm}^{-3}$ is the density of the PS particles, $R=$ $45 \mathrm{~nm}$ is the radius of the PS, and $w_{\text {core }}=0.77$ is the mass fraction of the PS core from a SPB particle obtained by the mass balance after purification.

The adsorption isotherm obtained from the ITC data is shown in Fig. 5(a) (circles). These data demonstrate the strong affinity of BLG towards the brush layer of the SPB. Most of the protein molecules are adsorbed from the aqueous solution
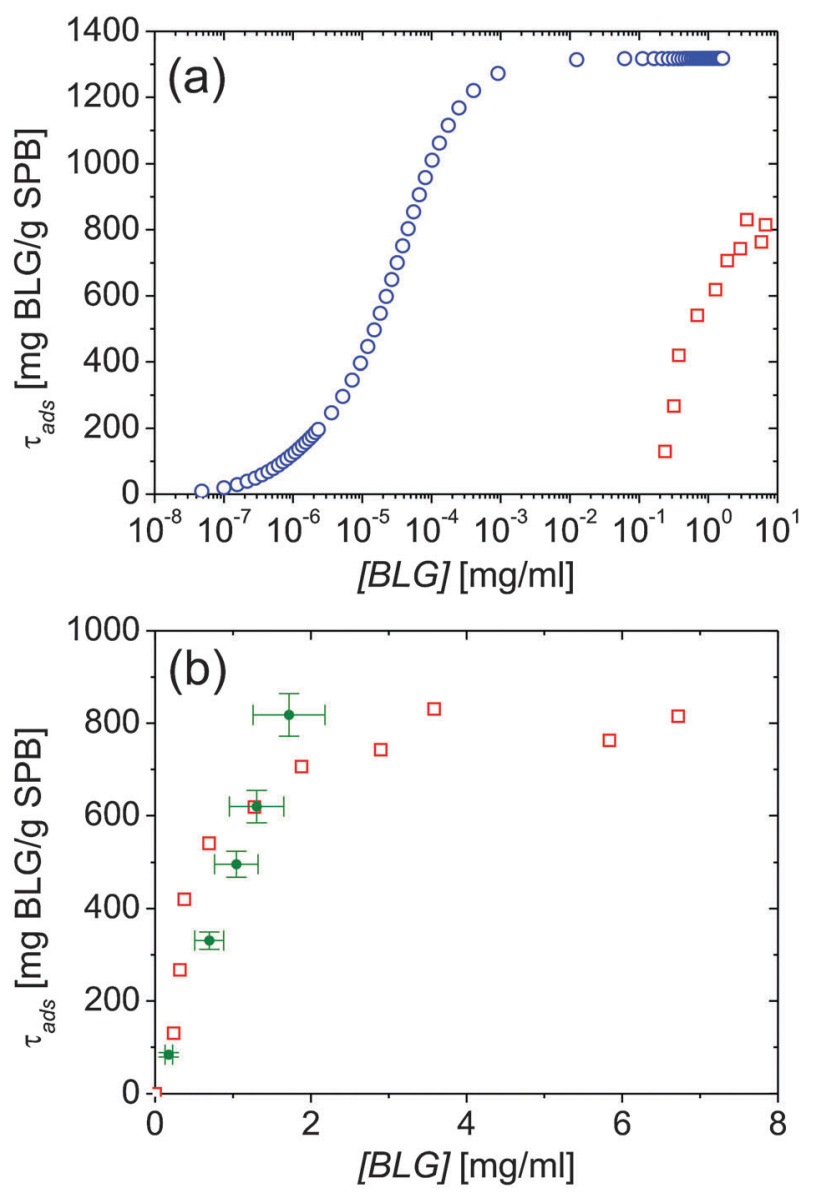

Fig. 5 (a) Comparison of adsorption isotherms obtained by different analytical methods (circles: ITC; squares: UF method). The UF method isotherm shows clearly that a significant amount of BLG can be removed by ultrafiltration. (b) Comparison of the SPB loading after ultrafiltration (squares) and by SAXS calculated protein loading of the inner part of the polyelectrolyte shell (filled circles).

until the full loading of the particles is reached leading to more than $1.3 \mathrm{~g}$ BLG per gram of carrier particles. Clearly a comparison of this isotherm with the SAXS-isotherm discussed in the previous section would be interesting. In principle, this comparison is possible if a sufficient fraction of protein molecules remains outside of the particles in aqueous solution. Then the analysis of the SAXS-data at small $q$ gives the amount of bound protein while the intensity of the scattering intensity measured at high $q$ gives the total amount of proteins present in the system. The amount of non-bound proteins is too small to be detected by SAXS in the present experiment. Here, the ITC provides a higher accuracy for the determination of an adsorption isotherm (cf. Fig. 5(a)).

\subsection{Adsorption isotherm by ultrafiltration}

The fraction of tightly bound protein can be determined by the UF method. The protein is adsorbed first onto the SPB particles. Afterwards, the free and slightly bound proteins are removed by extensive ultrafiltration. ${ }^{13,32}$ The results obtained from ITC and UF presented in Fig. 5(a) differ considerably. From this difference it is evident that a significant amount of BLG can be washed out by UF. 
As shown above, SAXS provides the distribution of the proteins inside the brush layer (see discussion of eqn (7)). The analysis of the SAXS data has shown that most of the proteins are bound in the inner $33 \mathrm{~nm}$ of the brush layer (cf. Fig. 2(b)). Evidently, the adsorbed protein in the outer part of the brush layer will be bound less tightly. This part of the adsorbed protein might be washed out during UF. To verify this prediction the amount of bound protein in different intervals of the polyelectrolyte layer can be determined from the SAXS analysis. The amount of adsorbed protein in the inner $33 \mathrm{~nm}$ with an error of $\pm 2.5 \mathrm{~nm}$ can be calculated and compared with the results from the ultrafiltration. For the investigated protein concentrations in Fig. 2(a) the result in Fig. 5(b) demonstrates that this is found indeed. The parameter of the curve is the protein concentration which remains in solution. While the agreement is somehow accidental since the location of the used limits for calculation is arbitrary, the comparison of both sets of data shows that only the proteins deep inside the polyelectrolyte layer are bound tightly. Evidently, this finding must be related to the radial decrease of the chain density in a SPB. The present results show that the interaction of the proteins with perhaps several polyelectrolyte chains is strong and even extensive ultrafiltration cannot wash out the bound molecules. Concomitantly, the $\mathrm{pH}$ in this region is considerably smaller than outside and the BLG starts to aggregate. In the outer region of the brush layer, on the other hand, the spatial density of the polyelectrolyte chains has become much smaller. Here, the binding of proteins resembles more the interaction of single polyelectrolyte chains with proteins. The comparison of SAXS, UF and ITC clearly shows that this binding is too weak to withstand prolonged ultrafiltration.

\section{Conclusions}

The adsorption of $\beta$-lactoglobulin onto SPB was investigated by SAXS at low ionic strength $(10 \mathrm{mM})$. The quantity and distribution of the adsorbed protein inside the polyelectrolyte shell can be determined by a core-shell model. The analysis of the data revealed that the adsorbed protein forms linear aggregates of about six monomer units inside the polyelectrolyte shell. Moreover, the SAXS allows us to analyze the amount of proteins bound at different radial positions of the SPB and compare this information to the binding isotherm obtained by ITC and ultrafiltration. This comparison (see Fig. 5) shows that proteins located in the inner regions of the SPB are tightly bound and cannot be washed away by UF whereas proteins bound in the outer layer are removed by UF.

\section{Acknowledgements}

We thank Financial support of the DFG, within ESFBIOSONS and Sonderforschungsbereich 840 is gratefully acknowledged. ESRF is thanked for provision of synchrotron beam time.

\section{References}

1 Physical Chemistry of Biological Interface, ed. A. Baskin and W. Norde, Marcel Dekker, New York, 1999.

2 W. Senaratne, L. Andruzzi and C. Ober, Biomacromolecules, 2005, 6, 2427.

3 D. B. Weibel and G. M. Whitesides, Curr. Opin. Chem. Biol., 2006, 10, 584.

4 L. M. A. E. Nel, D. Velegol, T. Xia, E. M. V. Hoek, P. Somasundaran, F. Klaessig, V. Castranova and M. Thompson, Nat. Mater., 2009, 8, 543.

5 M. Erol, H. Du and S. Sukhishvili, Langmuir, 2006, 22, 11329.

6 T. Cedervall, L. Iseult, S. Lindman, T. Berggard, E. Thulin, H. Nilsson, K. A. Dawson and S. Linse, Proc. Natl. Acad. Sci. U. S. A., 2007, 104, 2050.

7 S. Linse, C. Cabaleiro-Lago, W.-F. Xue, I. Lynch, S. Lindman, E. Thulin, S. E. Radford and K. A. Dawson, Proc. Natl. Acad. Sci. U. S. A., 2007, 104, 8691 .

8 C. Cabaleiro-Lago, F. Quinlan-Pluck, I. Lynch, S. Lindman, A. M. Minogue, E. Thulin, D. M. Walsh, K. A. Dawson and S. Linse, J. Am. Chem. Soc., 2008, 130, 15437.

9 S. Lindman, I. Lynch, E. Thulin, H. Nilsson, K. A. Dawson and S. Linse, Nano Lett., 2007, 4, 914.

10 M. Ballauff and O. Borisov, Curr. Opin. Colloid Interface Sci., 2006, 11, 316 .

11 P. Uhlmann, N. Houbenov, N. Brenner, K. Grundke, S. Burkert and M. Stamm, Langmuir, 2007, 23, 57.

12 O. Hollmann, R. Steitz and C. Czeslik, Phys. Chem. Chem. Phys., 2008, 10, 1448.

13 A. Wittemann and M. Ballauff, Phys. Chem. Chem. Phys., 2006, 8, 5269.

$14 \mathrm{~K}$. Henzler, A. Wittemann, E. Breininger, M. Ballauff and S. Rosenfeldt, Biomacromolecules, 2007, 8, 3674.

15 S. Rosenfeldt, A. Wittemann, M. Ballauff, E. Breininger, J. Bolze and N. Dingenouts, Phys. Rev. E: Stat., Nonlinear, Soft Matter Phys., 2004, 70, 061403.

16 K. Henzler, S. Rosenfeldt, A. Wittemann, L. Harnau, S. Finet, T. Narayanan and M. Ballauff, Phys. Rev. Lett., 2008, 100, 158301.

17 O. Hollmann and C. Czeslik, Langmuir, 2006, 22, 3300.

18 K. Henzler, B. Haupt, K. Lauterbach, A. Wittemann, O. Borisov and M. Ballauff, J. Am. Chem. Soc., 2010, 132, 3159.

19 M. T. Record, C. F. Anderson and T. M. Lohman, Q. Rev. Biophys., 1978, 11, 103.

20 C. Czeslik, G. Jackler, R. Steitz and H.-H. von Gruenberg, J. Phys. Chem. B, 2004, 108, 13395.

21 M. Ballauff, Prog. Polym. Sci., 2007, 32, 1135.

22 C. L. Cooper, P. L. Dubin, A. B. Kayitmazer and S. Turksen, Curr. Opin. Colloid Interface Sci., 2005, 10, 52.

23 C. Reichhart and C. Czeslik, Langmuir, 2009, 25, 1047.

24 A. Wittemann and M. Ballauff, Anal. Chem., 2004, 76, 2813.

25 G. Jackler, A. Wittemann, M. Ballauff and C. Czeslik, Spectroscopy, 2004, 18, 289.

26 M. Verheul, J. S. Pedersen, S. P. F. M. Roefs and K. G. de Kruif, Biopolymers, 1999, 49, 11.

27 S. N. Timasheff and R. Townend, Nature, 1964, 203, 517.

28 J. Witz, S. N. Timasheff and V. Luzzati, J. Am. Chem. Soc., 1964, 86, 168.

29 G. Panick, R. Malessa and R. Winter, Biochemistry, 1999, 38, 6512.

30 P. R. Majhi, R. R. Ganta, R. P. Vanam, E. Seyrek, K. Giger and P. L. Dubin, Langmuir, 2006, 22, 9150.

31 H. A. McKenzie, Adv. Protein Chem., 1967, 22, 55.

32 A. Wittemann, B. Haupt and M. Ballauff, Phys. Chem. Chem. Phys., 2003, 5, 1671.

33 P. Panine, S. Finet, T. M. Weiss and T. Narayanan, Adv. Colloid Interface Sci., 2006, 127, 9.

34 L. Li, S. Rosenfeldt, L. Harnau and M. Ballauff, Phys. Rev. E. Stat., Nonlinear, Soft Matter Phys., 2005, 72, 051504.

35 L. Harnau and J.-P. Hansen, J. Chem. Phys., 2002, 116, 9051.

36 J. S. Pedersen and M. C. Gerstenberg, Macromolecules, 1996, 29, 1363.

37 G. Vestergars Jensen, Q. Shi, M. J. Hernansanz, C. L. P. Oliveira, G. R. Deen, K. Almdal and J. S. Pedersen, J. Appl. Crystallogr., 2011, 44, 473.

38 L.-N. Lin, A. B. Manson, R. C. Woodworth and J. F. Brandts, Biochemistry, 1991, 30, 11660. 